FZJ-IKP(TH)-1999-29

June 16, 2018

\title{
Generalized Pions in Dense QCD
}

\author{
Mannque Rho ${ }^{a, b}$ 巴, Andreas Wirzbac ${ }^{2}$ and Ismail Zahed ${ }^{b[}$ \\ a Service de Physique Théorique, CE Saclay, 91191 Gif-sur-Yvette, France \\ ${ }^{b}$ Department of Physics and Astronomy, SUNY-Stony-Brook, NY 11794, U.S.A. \\ ${ }^{c}$ FZ Jülich, Institut für Kernphysik (Theorie), D-52425 Jülich, Germany
}

\begin{abstract}
QCD superconductors in the color-flavor-locked (CFL) phase sustain light Goldstone modes (that will be referred to as generalized pions) that can be described as pairs of particle and/or hole excitations around a gapped Fermi surface. In weak coupling and to leading logarithm accuracy, their form factor, mass and decay constant can be evaluated exactly. These modes are found to satisfy an axial-Ward-identity, constraining the mass of the Goldstone modes in the CFL phase.
\end{abstract}

\footnotetext{
${ }^{1}$ E-mail: rho@spht.saclay.cea.fr

${ }^{2}$ E-mail: a.wirzba@fz-juelich.de

${ }^{3}$ E-mail: zahed@zahed.physics.sunysb.edu
} 


\section{Introduction}

Quantum chromodynamics (QCD) at high density, relevant to the physics of the early universe, compact stars and relativistic heavy ion collisions, is presently attracting a renewed attention from both nuclear and particle theorists. Following an early suggestion by Bailin and Love [四, it was recently stressed that at large quark density, diquarks could condense into a color superconductor [2], with novel phenomena.

At large density, quarks at the edge of the Fermi surface interact weakly, although the high degeneracy of the Fermi surface causes perturbation theory to fail. Particles can pair and condense at the edge of the Fermi surface leading to energy gaps. Particle-particle and hole-hole pairing (BCS effect) have been extensively studied recently [1, 2]. Particle-hole pairing at the opposite edges of the Fermi surface (Overhauser effect) [3] has also begun to receive some attention [4, 5, 6]. This is however favored only by a large number of colors [4, 5, 6], strong coupling (large gaps) or lower dimensions [6].

The QCD superconductor breaks color and flavor symmetry spontaneously. As a result, the ground state exhibits Goldstone modes that are either particle-hole excitations (ordinary pions) or particle-particle and hole-hole excitations (BCS pions) with a mass that vanishes in the chiral limit. Effective-Lagrangian approaches to QCD in the color-flavorlocked (CFL) phase have been discussed recently by some of us [7] using a nonlinear realization of spontaneously broken color-flavor symmetry, and others [8] using a linear realization with hidden gauge symmetry. Both descriptions are equivalent - if vector dominance is exact - due to the Stuckelberg mechanism [9]. In general, the effective Lagrangian approach provides a convenient description of the long-wavelength physics based on global flavorcolor symmetries, including flavor-color anomalies, but does not allow one to determine the underlying parameters of the effective Lagrangian. These parameters are important for a quantitative description of the bulk (thermodynamic and transport) properties of the QCD superconductor, including for instance the mass of the recently discussed superqualiton [7]. They can only be determined using a more microscopic description of the QCD superconductor.

In this letter, we will derive explicit expressions for the form factor, temporal and spatial decay constants and mass of the Goldstone modes in the weak coupling regime in the CFL phase, and refer to [7, 8] for the discussion of the general aspects of the effective Lagrangian. In section 2 we discuss the general features of the QCD superconductor with screening. In section 3, we discuss the bound state problem in the CFL phase, and derive explicit results for the Goldstone modes. In section 4, we derive a general axial-Wardidentity in the QCD superconductor, constraining the mass of the Goldstone modes in weak coupling. Our conclusions are given in section 5 . 


\section{QCD Superconductor}

In the QCD superconductor, the quarks are gapped. Their propagation is given in the Nambu-Gorkov formalism by the following matrix

$$
\mathbf{S}=-i\langle\boldsymbol{\Psi} \overline{\mathbf{\Psi}}\rangle=\left(\begin{array}{cc}
S_{11} & S_{12} \\
S_{21} & S_{22}
\end{array}\right)
$$

in terms of the two-component Nambu-Gorkov field $\boldsymbol{\Psi}=\left(\psi, \psi_{C}\right)$, where $\psi$ refers to quarks and $\psi_{C}(q)=C \bar{\psi}^{T}(-q)$ to charge conjugated quarks, respectively \#. According to Ref. [10, the entries of $\mathbf{S}(q)$ in the massless case read

$$
\begin{aligned}
& S_{11}(q)=-i\langle\psi(q) \bar{\psi}(q)\rangle=\left[\frac{\Lambda^{+}(\mathbf{q})}{q_{0}^{2}-\epsilon_{q}^{2}}+\frac{\Lambda^{-}(\mathbf{q})}{q_{0}^{2}-\bar{\epsilon}_{q}^{2}}\right]\left(\not 1-\mu \gamma^{0}\right), \\
& S_{12}(q)=-i\left\langle\psi(q) \bar{\psi}_{C}(q)\right\rangle=-\mathbf{M}^{\dagger}\left[\frac{G^{*}(q) \Lambda^{+}(\mathbf{q})}{q_{0}^{2}-\epsilon_{q}^{2}}+\frac{\bar{G}^{*}(q) \Lambda^{-}(\mathbf{q})}{q_{0}^{2}-\bar{\epsilon}_{q}^{2}}\right], \\
& S_{21}(q)=-i\left\langle\psi_{C}(q) \bar{\psi}(q)\right\rangle=\left[\frac{G(q) \Lambda^{-}(\mathbf{q})}{q_{0}^{2}-\epsilon_{q}^{2}}+\frac{\bar{G}(q) \Lambda^{+}(\mathbf{q})}{q_{0}^{2}-\bar{\epsilon}_{q}^{2}}\right] \mathbf{M}, \\
& S_{22}(q)=-i\left\langle\psi_{C}(q) \bar{\psi}_{C}(q)\right\rangle=\left(\not q+\mu \gamma^{0}\right)\left[\frac{\Lambda^{+}(\mathbf{q})}{q_{0}^{2}-\epsilon_{q}^{2}}+\frac{\Lambda^{-}(\mathbf{q})}{q_{0}^{2}-\bar{\epsilon}_{q}^{2}}\right] .
\end{aligned}
$$

Here $\epsilon_{q} \equiv \mp\left\{(|\mathbf{q}|-\mu)^{2}+\mathbf{M}^{\dagger} \mathbf{M}|G(q)|^{2}\right\}^{1 / 2} \approx \mp\left\{(|\mathbf{q}|-\mu)^{2}+|G(q)|^{2}\right\}^{1 / 2}$ are the energies of a particle/hole \#3, whereas the energies of an antiparticle/hole are given by $\bar{\epsilon}_{q} \approx$ $\mp\left\{(|\mathbf{q}|+\mu)^{2}+|\bar{G}(q)|^{2}\right\}^{1 / 2}[10,11]$. The particle and antiparticle gaps are denoted by the complex-valued functions $G(q)$ and $\bar{G}(q)$, respectively. The operators $\Lambda^{ \pm}(\mathbf{q})=\frac{1}{2}(1 \pm \boldsymbol{\alpha} \cdot \hat{\mathbf{q}})$ are the particle/antiparticle projectors The charge conjugation operator $C$ is already incorporated in the definition of the NambuGorkov field $\Psi$.

For large $\mu$, the antiparticles decouple: $q_{\|} \approx(|\mathbf{q}|-\mu)$ is the particle/hole momentum at the Fermi surface in the direction of the Fermi momentum, such that $\epsilon_{q} \approx \mp \sqrt{q_{\|}^{2}+|G(q)|^{2}}$ and $\bar{\epsilon}_{q} \approx \mp 2 \mu$. Therefore, we have

$$
\mathbf{S} \approx\left(\begin{array}{rc}
\gamma^{0}\left(q_{0}+q_{\|}\right) \Lambda^{-}(\mathbf{q}) & -\mathbf{M}^{\dagger} G^{*}(q) \Lambda^{+}(\mathbf{q}) \\
\mathbf{M} G(q) \Lambda^{-}(\mathbf{q}) & \gamma^{0}\left(q_{0}-q_{\|}\right) \Lambda^{+}(\mathbf{q})
\end{array}\right) \frac{1}{q_{0}^{2}-\epsilon_{q}^{2}}
$$

with $q_{0}^{2}-\epsilon_{q}^{2} \approx q_{0}^{2}-q_{\|}^{2}-|G(q)|^{2}$. Using the color-identity

$$
\sum_{a} \frac{\lambda^{a T}}{2} \epsilon^{c} \frac{\lambda^{a}}{2}=-\frac{4}{6} \epsilon^{c},
$$

${ }^{\# 1} q=\left(q^{0}, \mathbf{q}\right)$ and $\bar{\psi}^{T}$ is the transposed and conjugated field with $C \equiv i \gamma^{2} \gamma^{0}$.

${ }^{\# 2}$ We are adopting the standard phase convention between $\langle\psi \bar{\psi}\rangle$ and $S(q)$.

${ }^{\# 3}$ This approximation assumes $\mathbf{M}^{\dagger} \mathbf{M} \approx \mathbf{1}_{c f}$ in the mass-shell condition.

${ }^{\# 4}$ Note that $\gamma^{0} \Lambda^{ \pm}(\mathbf{q})=\Lambda^{\mp}(\mathbf{q}) \gamma^{0}, \gamma^{5} \Lambda^{ \pm}(\mathbf{q})=\Lambda^{ \pm}(\mathbf{q}) \gamma^{5}$ and $\boldsymbol{\alpha} \cdot \hat{\mathbf{q}} \Lambda^{ \pm}(\mathbf{q})= \pm|\mathbf{q}| \Lambda^{ \pm}(\mathbf{q})$. 

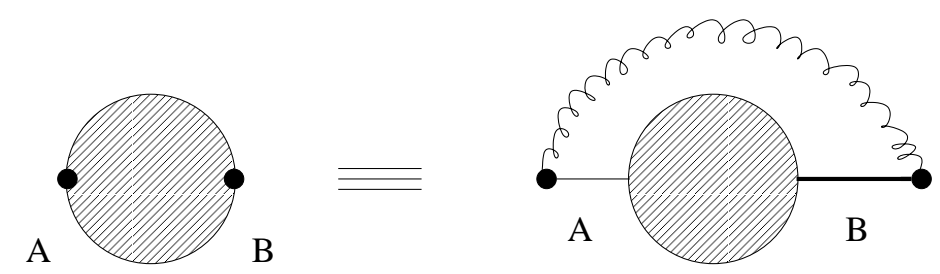

Figure 1: BCS gap equation. The thin and thick lines are the free and dressed quark propagator, respectively, whereas the wiggly line is the gluon propagator with A, B as Nambu-Gorkov indices.

the gap function $G(q)$ in the CFL phase satisfies (see Fig. 1)

$$
\begin{aligned}
G(p) & =\frac{4 g^{2}}{3} \int \frac{d^{4} q}{(2 \pi)^{4}} i \mathcal{D}(p-q) \frac{G(q)}{q_{0}^{2}-\epsilon_{q}^{2}} \\
& =\frac{4 g^{2}}{3} \int \frac{d^{4} q_{E}}{(2 \pi)^{4}} \mathcal{D}(p-q) \frac{G(q)}{q_{4}^{2}+q_{\|}^{2}+|G(q)|^{2}} .
\end{aligned}
$$

The second expression refers to Euclidean coordinates. We note that a similar equation is fulfilled by the antiparticle gaps through $G(p) \rightarrow \bar{G}(p)$ on the left hand side of (5) for the present approximations. For perturbative screening, the gluon-propagator in Euclidean space reads

$$
\mathcal{D}(q)=\frac{1}{2} \frac{1}{q^{2}+m_{E}^{2}}+\frac{1}{2} \frac{1}{q^{2}+m_{M}^{2}} .
$$

Perturbative arguments give $m_{E}^{2} /(g \mu)^{2}=m_{D}^{2} /(g \mu)^{2} \approx N_{f} / 2 \pi^{2}$ and $m_{M}^{2} / m_{D}^{2} \approx \pi\left|q_{4}\right| /|4 \mathbf{q}|$, where $m_{D}$ is the Debye mass, $m_{M}$ is the magnetic screening generated by Landau damping and $N_{f}$ the number of flavors [12]. Throughout we will refer to $m_{M}$ loosely as the magnetic screening mass. We note that the perturbative screening vanishes at large $N_{c}$. Nonperturbative arguments for screening [13] will not be addressed here.

For a constant gap, (5) diverges logarithmically. This is an ultraviolet effect that should not affect the infrared behaviour at the Fermi surface [11]. With this in mind, we obtain

$$
\begin{aligned}
G\left(p_{\|}\right) \approx \frac{h_{*}^{2}}{6} \int_{0}^{\infty} & d q_{\|} \frac{G\left(q_{\|}\right)}{\sqrt{q_{\|}^{2}+\left|G\left(q_{\|}\right)\right|^{2}}} \\
& \times \ln \left\{\left(1+\frac{\Lambda_{\perp}^{2}}{\left(p_{\|}-q_{\|}\right)^{2}+m_{E}^{2}}\right)^{3}\left(1+\frac{\Lambda_{\perp}^{3}}{\left|p_{\|}-q_{\| \mid}\right|^{3}+\frac{\pi}{4} m_{D}^{2}\left|p_{\|}-q_{\|}\right|}\right)^{2}\right\},
\end{aligned}
$$

where

$$
h_{*}^{2}=\frac{4}{3} \frac{g^{2}}{8 \pi^{2}} .
$$

${ }^{\# 5}$ We are using a simplified version as in $[6$. To leading logarithm accuracy, the results are unaffected. 
These relations can be readily generalized to arbitrary $N_{c}, N_{f}$ in the CFL phase [6]. The transverse cutoff $\Lambda_{\perp}=2 \mu$ is exactly fixed in weak coupling. Hence $\Lambda_{\perp}>m_{E}, m_{M}$ and the logarithm in (7) may not be expanded. To leading logarithm accuracy, the gap equation (5) can be solved using $x=\ln \left(\Lambda_{*} / p\right)$ with $\Lambda_{*}=\left(4 \Lambda_{\perp}^{6} / \pi m_{E}^{5}\right)$ in the weak coupling limit. The result is

$$
G(x)=G_{0} \sin \left(h_{*} x / \sqrt{3}\right)
$$

with $G_{0}$ given by

$$
G_{0} \approx\left(\frac{4 \Lambda_{\perp}^{6}}{\pi m_{E}^{5}}\right) e^{-\frac{\sqrt{3} \pi}{2 h_{*}}} .
$$

This result is the same as the one reached in [14, 11, 15, 6]. We further note that the chiral condensate vanishes in the chiral limit,

$$
\langle\bar{\Psi} \boldsymbol{\Psi}\rangle=i \int \frac{d^{4} q}{(2 \pi)^{4}} \operatorname{Tr} \mathbf{S}(q)=0
$$

because of the vector character of the interaction at the gap, see e.g. (3). However, the composite (quartic) chiral condensates do not, e.g.

$$
\left\langle(\overline{\mathbf{\Psi}} \boldsymbol{\Psi})^{2}\right\rangle=\int \frac{d^{4} q}{(2 \pi)^{4}} \operatorname{Tr}\left[\mathbf{S}(q) \mathbf{S}^{\dagger}(q)\right] \neq 0
$$

\section{Pions in the CFL Phase}

At high density, QCD with $N_{f}=N_{c}=3$ and three degenerate light quarks exhibits a phase with color-flavor locking that is multiply degenerate [16], i.e.

$$
\left\langle\overline{\mathbf{\Psi}} \mathbf{M}^{a \alpha}\left(e^{-i \gamma_{5} \pi^{A} \mathbf{T}^{A}}\right)^{a \alpha} \rho_{2} \boldsymbol{\Psi}\right\rangle \neq 0
$$

with $\mathbf{M}^{a \alpha}=\epsilon_{f}^{a} \epsilon_{c}^{\alpha} \gamma_{5}=\left(\mathbf{M}^{\dagger}\right)^{a \alpha}, \rho_{2}$ a Pauli matrix active on the Nambu-Gorkov entries, and $\mathbf{T}^{A}=\operatorname{diag}\left(\tau^{A}, \tau^{A^{*}}\right)$ an $S U(3)_{c+F}$ valued generator in the Nambu-Gorkov representation. The CFL phase is invariant under the diagonal of rigid vector-color plus vector-flavor, i.e. $S U(3)_{c+V}$. The Goldstone modes in the CFL phase can be regarded as excitations with particle and/or hole content. Their wavefunction is driven by the Bethe-Salpeter kernel shown in Fig. 2. Specifically, \#5

$$
\boldsymbol{\Gamma}^{\alpha}(p, P)=g^{2} \int \frac{d^{4} q}{(2 \pi)^{4}} i \mathcal{D}(p-q) i \mathbf{V}_{\mu}^{a} i \mathbf{S}\left(q+\frac{P}{2}\right) \boldsymbol{\Gamma}^{\alpha}(q, P) i \mathbf{S}\left(q-\frac{P}{2}\right) i \mathbf{V}_{a}^{\mu}
$$

\footnotetext{
${ }^{\# 6}$ Note that the Fermion propagators are directed. Therefore, the two propagators incorporate the total momentum $P$ with opposite sign and the relative momentum $q$ with the same sign.
} 

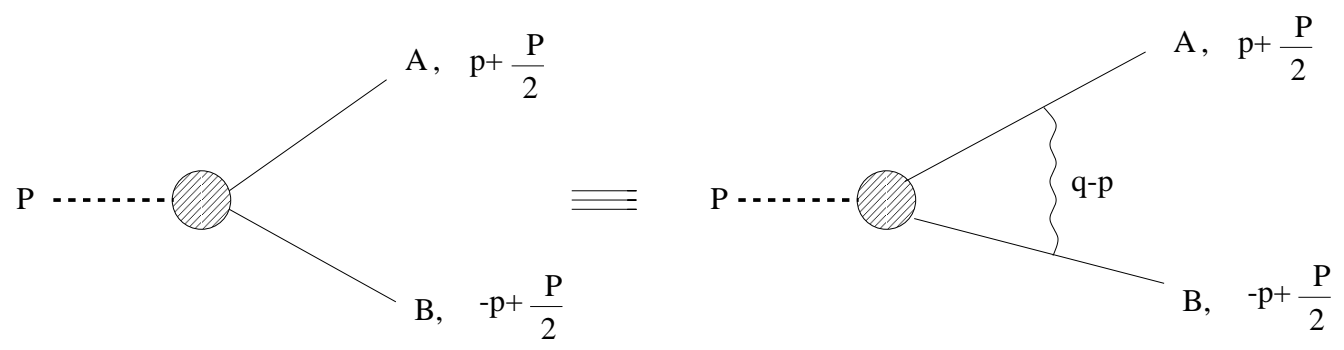

Figure 2: Bethe-Salpeter equation for the pions in the QCD superconductor.

and the vertex is defined as

$$
\mathbf{V}_{\mu}^{a} \equiv\left(\begin{array}{cc}
\gamma_{\mu} \lambda^{a} / 2 & 0 \\
0 & C\left(\gamma_{\mu} \lambda^{a} / 2\right)^{T} C^{-1}
\end{array}\right)=\left(\begin{array}{cc}
\gamma_{\mu} \lambda^{a} / 2 & 0 \\
0 & -\gamma_{\mu} \lambda^{a T} / 2
\end{array}\right)
$$

For $P=0$, the Bethe-Salpeter equation (14) admits the following solution for the CFL pion vertex

$$
\boldsymbol{\Gamma}^{A}(p, 0)=i \gamma_{5} \frac{1}{F}\left(\begin{array}{cc}
0 & -G^{*}(p) \mathbf{M}^{A^{\dagger}} \\
G(p) \mathbf{M}^{A} & 0
\end{array}\right)
$$

with $\mathbf{M}^{A}=\mathbf{M}^{a \alpha}\left(\tau^{A}\right)^{a \alpha}$ and $\mathbf{M}^{A^{\dagger}}=\mathbf{M}^{a \alpha}\left(\tau^{A^{*}}\right)^{a \alpha}$. Indeed, in terms of (16) the BetheSalpeter equation for $P=0$ reduces to (14) with (8) for $h_{*}^{2}$, after using

$$
\sum_{a} \frac{\lambda^{a T}}{2}\left(\mathbf{M}^{A}\right) \frac{\lambda^{a}}{2}=-\frac{2}{3} \mathbf{M}^{A}, \quad \sum_{a} \frac{\lambda^{a T}}{2}\left(\mathbf{M} \mathbf{M}^{A^{\dagger}} \mathbf{M}\right) \frac{\lambda^{a}}{2} \approx-\frac{2}{3} \mathbf{M}^{A}
$$

and ignoring the symmetric contributions in color-flavor which are subleading in leading logarithm accuracy. Hence $G(p)=G^{*}(p)$ satisfies the gap equation (5) and $F$ can be identified as the pion decay constant. The distinction between temporal $F_{T}$ and spatial $F_{S}$ will be made below. We note that $G(p)$ in the vertex (16) plays the role of the pion form factor in weak coupling in the CFL phase. In the effective Lagrangian approach [0, 8] this feature is usually ignored by treating the pions as point-like.

Having identified the Goldstone modes, we now proceed to determine the pion decay constant $F$. For that we use the standard definition in terms of the axial vector current $\#$

$$
\left\langle\operatorname{BCS}\left|\mathbf{A}_{\mu}^{\alpha}(0)\right| \pi_{B}^{\beta}(P)\right\rangle \equiv i F P_{\mu} \delta^{\alpha \beta}
$$

In terms of the original quark fields $\psi$, the axial vector current follows from Noether theorem. Hence

$$
\mathbf{A}_{\mu}^{\alpha}(x)=\overline{\mathbf{\Psi}} \gamma_{\mu} \gamma_{5} \frac{1}{2} \mathbf{T}^{\alpha} \mathbf{\Psi}
$$

\footnotetext{
${ }^{\# 7}$ Note that because of the common term $+P_{\mu}$, the charged conjugated field in momentum space transforms as $\psi_{C}(q)=C \bar{\psi}^{T}(-q)$, although the corresponding field in position space transforms just as $\psi_{C}(x)=C \bar{\psi}^{T}(+x)$; see Ref. 10].
} 
In terms of $(16)$ and $(19)$ the relation (18) is given by the diagrams in Fig. 3. Specifically,

$$
i F P_{\mu} \delta^{\alpha \beta}=-\int \frac{d^{4} k}{(2 \pi)^{4}} \operatorname{Tr}\left(\gamma_{\mu} \gamma_{5} \frac{1}{2} \mathbf{T}^{\alpha} i \mathbf{S}\left(k+\frac{P}{2}\right) i \boldsymbol{\Gamma}^{\beta}(k, P) i \mathbf{S}\left(k-\frac{P}{2}\right)\right) .
$$

In the (massive) pion rest frame $P=(M, 0)$, (20) can be unwound for the temporal component of the axial-vector current. Expanding the right-hand-side of (20) to leading order in $M$, yields the temporal pion decay constant

$$
F_{T}^{2} \approx-8 i \int \frac{d^{4} k}{(2 \pi)^{4}} \frac{|G(k)|^{2}}{\left(k_{0}^{2}-\epsilon_{k}^{2}\right)^{2}} .
$$

The result (21) is reminiscent of the result for the Goldstone modes in the normal phase obtained with the substitution $G(p) \rightarrow M(p)$ (constituent mass) [17]. In the normal phase (with $\mu=0$ ), Lorentz symmetry is intact, so $F_{T}=F_{S}$. At finite $\mu$, Lorentz symmetry is upset 18, 19, 20]. Indeed, the spatial component of (20) yields instead

$$
F_{S}^{2} \approx-8 i \int \frac{d^{4} k}{(2 \pi)^{4}} \frac{|G(k)|^{2}}{\left(k_{0}^{2}-\epsilon_{k}^{2}\right)^{2}}(\hat{\mathbf{k}} \cdot \hat{\mathbf{P}})^{2}=\frac{1}{3} F_{T}^{2} .
$$

In the CFL phase the Goldstone modes travel at a speed less than the speed of light. The factor of $1 / 3$ in (22) is easily understood as the average of the current direction squared over the Fermi surface.

For a constant gap, (21) diverges logarithmically. However, this is an ultraviolet effect similar to the one already observed in the gap equation 11] that can be subtracted without affecting $F$ at the Fermi surface at least to leading logarithm accuracy. Assuming $G(k) \equiv G\left(k_{\|}\right)$, and performing the integration over $k_{0}$ by contour with $\epsilon_{k} \rightarrow \epsilon_{k}-i \epsilon$, we obtain

$$
F_{T}^{2} \approx \frac{2 \mu^{2}}{\pi^{2}} \int_{0}^{\infty} d k_{\|} \frac{\left|G\left(k_{\|}\right)\right|^{2}}{\epsilon_{k}^{3}} .
$$

Inserting the leading logarithm solution (9) to the screened gap equation (7) in (23) and defining $x_{0}=\ln \left(\Lambda_{*} / G_{0}\right)=\sqrt{3} \pi /\left(2 h_{*}\right)$, we have

$$
F_{T}^{2} \approx \frac{2 \mu^{2}}{\pi^{2}} \int_{0}^{x_{0}} d x e^{2\left(x-x_{0}\right)} \sin ^{2}\left(\frac{\pi x}{2 x_{0}}\right)=\frac{\mu^{2}}{2 \pi^{2}} \frac{8 x_{0}^{2}+\pi^{2}\left(1-e^{-2 x_{0}}\right)}{4 x_{0}^{2}+\pi^{2}} \approx \frac{\mu^{2}}{\pi^{2}} .
$$

Hence, $F_{T}^{2} / G_{0}^{2} \gg 1$, implying that the 'size' of the pions $r_{\pi} \approx 1 / F_{T}$ in the CFL phase is very small. The inverse size of the pion relates to the inverse transverse momentum exchanged between pairs at the Fermi surface, which is of order $\mu$, irrespective of screening. Clearly $F_{T}$ vanishes if the BCS gap vanishes through (21).

\section{Axial Ward-identity}




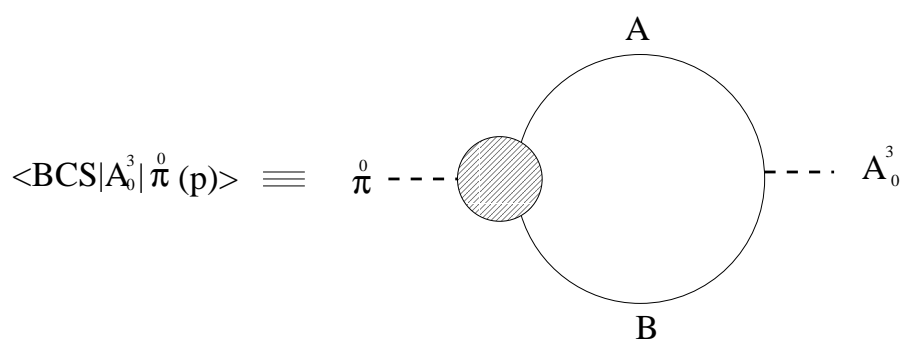

Figure 3: Axial-vector transition in the QCD superconductor.

The underlying flavor symmetry of the QCD action entails chiral Ward identities in the QCD superconductor with relations between the mass and decay constant of the Goldstone modes. Indeed, when chiral symmetry is explicitly broken by massive quarks $m_{f}=\left(m_{u}, m_{d}, m_{s}\right)$, then the pions are expected to be massive. Hence

$$
0 \equiv \int d^{4} x \partial_{x}^{\mu}\left\langle\operatorname{BCS}\left|T^{*} \mathbf{A}_{\mu}^{\alpha}(x) \boldsymbol{\pi}_{B}^{\beta}(0)\right| \mathrm{BCS}\right\rangle
$$

where the axial-vector current $\mathbf{A}_{\mu}^{a}$ is given in (19) and the pion field $\boldsymbol{\pi}_{B}(x)$ in the QCD superconductor is defined as

$$
\boldsymbol{\pi}_{B}^{\beta}(x)=\left(\begin{array}{cc}
0 & \bar{\psi} \gamma^{0}\left(i \tau^{\beta} \gamma_{5} \mathbf{M}\right)^{\dagger} \gamma^{0} \psi_{C}(x) \\
\bar{\psi}_{C} \mathbf{M} i \tau^{\beta} \gamma_{5} \psi(x) & 0
\end{array}\right) .
$$

The flavor axial-vector current in the CFL phase obeys the local divergence equation

$$
\partial \cdot \mathbf{A}^{\alpha}(x)=\left(\begin{array}{cc}
\bar{\psi} i \frac{1}{2}\left[m_{f}, \tau^{\alpha}\right]_{+} \gamma_{5} \psi(x) & 0 \\
0 & \bar{\psi}_{C} i \frac{1}{2}\left[m_{f}, \tau^{\alpha *}\right]_{+} \gamma_{5} \psi_{C}(x)
\end{array}\right)
$$

For massless quarks, the hermitean axial-isovector charge

$$
\mathbf{Q}_{5}^{\alpha} \equiv \mathbf{Q}_{5}^{\alpha}\left(x^{0}\right)=\int d^{3} x \operatorname{Tr}\left(\begin{array}{cc}
\bar{\psi} \frac{1}{2} \tau^{\alpha} \gamma^{0} \gamma_{5} \psi(x) & 0 \\
0 & \bar{\psi}_{C} \frac{1}{2} \tau^{\alpha *} \gamma^{0} \gamma_{5} \psi_{C}(x)
\end{array}\right)
$$

is conserved and generates axial-vector rotations, e.g.

$$
\left[\mathbf{Q}_{5}^{\alpha}, \mathbf{\Psi}(x)\right]=i \gamma_{5} \frac{1}{2} \mathbf{T}^{\alpha} \mathbf{\Psi}(x) .
$$

In terms of (2729), the identity (26) yields the axial Ward-identity

$$
\int d^{4} x\left\langle\operatorname{BCS}\left|T^{*} \frac{1}{2}\left[m_{f}, \boldsymbol{\pi}^{\alpha}(x)\right]_{+} \boldsymbol{\pi}_{B}^{\beta}(0)\right| \operatorname{BCS}\right\rangle=\left\langle\operatorname{BCS}\left|\boldsymbol{\Sigma}_{B}^{\alpha \beta}(0)\right| \operatorname{BCS}\right\rangle,
$$

where the diquark field $\boldsymbol{\Sigma}_{B}^{\alpha \beta}(x)$ is defined as

$$
\boldsymbol{\Sigma}_{B}^{\alpha \beta}(x)=\left(\begin{array}{cc}
0 & \bar{\psi} \gamma^{0}\left[\frac{1}{2} \tau^{\alpha}, \mathbf{M}^{\dagger} \tau^{\beta}\right]_{+} \gamma^{0} \psi_{C}(x) \\
\bar{\psi}_{C}\left[\frac{1}{2} \tau^{\alpha}, \mathbf{M} \tau^{\beta}\right]_{+} \psi(x) & 0
\end{array}\right)
$$


and $\boldsymbol{\pi}(x)$ is the diagonal pion field

$$
\boldsymbol{\pi}^{\alpha}(x)=\left(\begin{array}{cc}
\bar{\psi} i \tau^{\alpha} \gamma_{5} \psi(x) & 0 \\
0 & \bar{\psi}_{C} i \tau^{\alpha *} \gamma_{5} \psi_{C}(x)
\end{array}\right)
$$

The latter is to be contrasted with the off-diagonal or BCS pion field (26). Clearly in the QCD superconductor, $\boldsymbol{\pi}(x)$ and $\boldsymbol{\pi}_{B}(x)$ mix through (30). This is expected, since particles and/or holes can pop up from the superconducting state, thereby changing a normal pion to a BCS pion. The true pion is a linear combination of both, and the number of pseudoscalar Goldstone modes is only commensurate with the dimension of the manifold spanned by (13). A typical contribution to (30) is shown in Fig. \$a and $4 \mathrm{~b}$. The dotted insertion in Fig. Gb corresponds to the BCS pion exchange in the superconductor. The nonconfining character of the weak coupling description allows for the occurrence of the gapped $q q$ and/or $\bar{q} q$ exchange of Fig. 国a. Hence,

$$
\begin{gathered}
\left\langle\operatorname{BCS}\left|\boldsymbol{\Sigma}_{B}^{\alpha \beta}(0)\right| \operatorname{BCS}\right\rangle \approx-\int \frac{d^{4} q}{(2 \pi)^{4}} \operatorname{Tr}\left[i \gamma_{5} \frac{1}{2}\left[m_{f}, \mathbf{T}^{\alpha}\right]_{+} i \mathbf{S}(q) \mathbf{\Pi}_{B}^{\beta} i \mathbf{S}(q)\right] \\
-\left\{\int \frac{d^{4} q}{(2 \pi)^{4}} \operatorname{Tr}\left[i \gamma_{5} \frac{1}{2}\left[m_{f}, \mathbf{T}^{\alpha}\right]_{+} i \mathbf{S}(q) i \boldsymbol{\Gamma}^{\xi} i \mathbf{S}(q)\right]\right\}\left(\frac{i}{M^{2}}\right)^{\xi \xi^{\prime}}\left\{\int \frac{d^{4} q}{(2 \pi)^{4}} \operatorname{Tr}\left[i \boldsymbol{\Gamma}^{\xi^{\prime}} i \mathbf{S}(q) \boldsymbol{\Pi}_{B}^{\beta} i \mathbf{S}(q)\right]\right\}
\end{gathered}
$$

with

$$
\mathbf{\Pi}_{B}^{\beta} \equiv\left(\begin{array}{cc}
0 & \gamma^{0}\left(i \tau^{\beta} \gamma_{5} \mathbf{M}\right)^{\dagger} \gamma^{0} \\
i \tau^{\beta} \gamma_{5} \mathbf{M} & 0
\end{array}\right)
$$

In the chiral limit $m_{i} \rightarrow 0, i \in\{u, d, s\}$, the first term in (33) (Fig. Ga) drops out and the identity is fulfilled if $1 / M^{2}$ is sufficiently singular in $m_{i}$ to match the numerator. The traces can be evaluated in weak coupling. The result is \#8

$$
\begin{aligned}
& \int \frac{d^{4} q}{(2 \pi)^{4}} \operatorname{Tr}\left[i \gamma_{5} \frac{1}{2}\left[m_{f}, \mathbf{T}^{\alpha}\right]_{+} i \mathbf{S}(q) i \boldsymbol{\Gamma}^{\xi} i \mathbf{S}(q)\right]=\mathcal{O}\left(m_{f}^{2}\right), \\
& \int \frac{d^{4} q}{(2 \pi)^{4}} \operatorname{Tr}\left[i \boldsymbol{\Gamma}^{\xi^{\prime}} i \mathbf{S}(q) \mathbf{\Pi}_{B}^{\beta} i \mathbf{S}(q)\right]=\delta^{\xi^{\prime} \beta} \frac{16 i}{F_{T}} \int \frac{d^{4} q}{(2 \pi)^{4}} \frac{G(q)}{q_{0}^{2}-\epsilon_{q}^{2}},
\end{aligned}
$$

which shows that $M^{2}=\mathcal{O}\left(m_{f}^{2}\right)$. To determine the coefficient, we need to expand the vertices and the propagators in (33) to leading order in $m_{f}$. The $\mathcal{O}\left(m_{f}\right)$ corrections to both $G(p)$ and $\Gamma(p)$ do not contribute. They trace to zero because of a poor spin structure. Therefore, only the $\mathcal{O}\left(m_{f}\right)$ correction to the propagator (2) is needed, i.e.

$$
\Delta \mathbf{S}(q) \approx\left(\begin{array}{cc}
\frac{m_{f}}{2 \mu} \frac{q_{0}+q_{\|}}{q_{0}^{2}-\epsilon_{q}^{2}} & \gamma^{0}\left(\frac{m_{f} \mathbf{M}^{\dagger} \Lambda^{+}(\mathbf{q})}{2 \mu}+\frac{\mathbf{M}^{\dagger} m_{f} \Lambda^{-}(\mathbf{q})}{2 \mu}\right) \frac{G^{*}(q)}{q_{0}^{2}-\epsilon_{q}^{2}} \\
\gamma^{0}\left(\frac{m_{f} \mathbf{M} \Lambda^{-}(\mathbf{q})}{2 \mu}+\frac{\mathbf{M} m_{f} \Lambda^{+}(\mathbf{q})}{2 \mu}\right) \frac{G(q)}{q_{0}^{2}-\epsilon_{q}^{2}} & \frac{m_{f}}{2 \mu} \frac{-q_{0}+q_{\|}}{q_{0}^{2}-\epsilon_{q}^{2}}
\end{array}\right) .
$$

\footnotetext{
${ }^{\# 8}$ The use of $F_{T}$ instead of $F_{S}$ in the pion vertex follows from the fact that the intermediate BCS pion is generated by a chiral rotation of the BCS ground state. A similar interpretation in matter is made in 19 .
} 


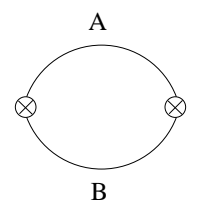

(a)

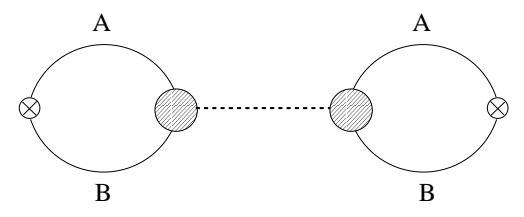

(b)

Figure 4: (a) connected and (b) disconnected (generalized pion) contribution in the QCD superconductor.

Using (36) yields for the first trace in (35)

$$
\begin{aligned}
& \int \frac{d^{4} q}{(2 \pi)^{4}} \operatorname{Tr}\left[i \gamma_{5} \frac{1}{2}\left[m_{f}, \mathbf{T}^{\alpha}\right]_{+} i \mathbf{S}(q) i \boldsymbol{\Gamma}^{\xi} i \mathbf{S}(q)\right] \\
& =\frac{\mu G_{0}}{8 \pi^{2} F_{T}} \operatorname{Tr}_{c f}\left(\left[m_{f}^{2}, \tau^{\alpha}\right]\left(\mathbf{M}^{\dagger} \mathbf{M}^{\beta}-\mathbf{M}^{\beta^{\dagger}} \mathbf{M}\right)+\left[m_{f}^{2}, \tau^{\alpha *}\right]\left(\mathbf{M M}^{\beta^{\dagger}}-\mathbf{M}^{\beta} \mathbf{M}^{\dagger}\right)\right)
\end{aligned}
$$

Using (35) and (37) in (33) and noting that

$$
\left\langle\Sigma_{B}^{\alpha \beta}\right\rangle \equiv \operatorname{Tr}\left(\left[\frac{\tau^{\alpha}}{2}, \mathbf{M} \tau^{\beta}\right]_{+} \rho_{2} \mathbf{S}\right)=\delta^{\alpha \beta} 8 i \int \frac{d^{4} q}{(2 \pi)^{4}} \frac{G(q)}{q_{0}^{2}-\epsilon_{q}^{2}}
$$

we obtain for the mass of the Goldstone modes

$$
\begin{gathered}
\left(M^{2}\right)^{\alpha \beta} \approx \frac{\mu G_{0}}{4 \pi^{2} F_{T}^{2}} \operatorname{Tr}_{c f}\left(\left[m_{f}^{2}, \tau^{\alpha}\right]\left(\mathbf{M}^{\dagger} \mathbf{M}^{\beta}-\mathbf{M}^{\beta^{\dagger}} \mathbf{M}\right)+\left[m_{f}^{2}, \tau^{\alpha *}\right]\left(\mathbf{M M}^{\beta^{\dagger}}-\mathbf{M}^{\beta} \mathbf{M}^{\dagger}\right)\right) \\
\approx \sqrt{\frac{2}{3}} \frac{256 \pi^{4}}{9 g^{5}} \exp \left(-\frac{3 \pi^{2}}{\sqrt{2} g}\right)\left\{\operatorname{Tr}_{c f}\left(\left[m_{f}^{2}, \tau^{\alpha}\right]\left(\mathbf{M}^{\dagger} \mathbf{M}^{\beta}-\mathbf{M}^{\beta^{\dagger}} \mathbf{M}\right)\right)\right. \\
\left.\quad+\operatorname{Tr}_{c f}\left(\left[m_{f}^{2}, \tau^{\alpha *}\right]\left(\mathbf{M M}^{\beta^{\dagger}}-\mathbf{M}^{\beta} \mathbf{M}^{\dagger}\right)\right)\right\}
\end{gathered}
$$

where (24) and (10) were inserted into the last line for $F_{T}^{2}$ and $G_{0}$, respectively. Furthermore, $m_{E}=\sqrt{(3 / 2)} g \mu / \pi$ was used. The $m_{f}^{2}$ behaviour is consistent with the one suggested in [21. The color-flavor trace in (39) vanishes, suggesting that the generalized pions remain massless to order $m_{f}^{2}$ in the CFL phase $\#$.

\section{Conclusions}

We have discussed certain bulk features of the QCD superconductor. In the CFL phase, the order parameter is multidegenerate leading to Goldstone modes, with temporal and spatial decay constants that can be calculated exactly in weak coupling. We find

\footnotetext{
\#9 This result is exact in mass perturbation theory in the CFL phase modulo footnote 3.
} 
$F_{T}^{2} / G_{0}^{2} \gg 1$ and $F_{S}^{2} / F_{T}^{2}=1 / 3$. The Goldstone modes have a very small size and propagate with a speed that is less than the speed of light. The multidegeneracy of the Goldstone manifold is lifted by finite quark masses. The Goldstone modes are found to obey a generalized axial Ward identity, constraining the mass of the pion in the CFL phase. We note that the small size of the pion implies that the recently discussed superqualitons [7] are in general heavy, with $M_{s} / G_{0} \approx\left(F_{S} / G_{0}\right)^{2} \gg 1$. The mismatch between the temporal and spatial decay constants may be relevant for soft pion emission in cold and dense matter. Further issues regarding the CFL spectrum will be discussed elsewhere.

\section{Acknowledgments}

IZ thanks Edward Shuryak for discussions. We thank Y. Kim for help with the Figures. This work was supported in part by US-DOE DE-FG-88ER40388 and DE-FG02-86ER40251.

Note Added: After completion of our work Ref. 29] appeared where similar issues were addressed using different arguments.

\section{References}

[1] D. Bailin and A. Love, Phys. Rept. 107, 325 (1984).

[2] M. Alford, K. Rajagopal and F. Wilczek, Phys. Lett. B422, 247 (1998), hep-ph/9711395; R. Rapp, T. Schäfer, E.V. Shuryak and M. Velkovsky, Phys. Rev. Lett. 81, 53 (1998), hep-ph/9711396; T. Schäfer, Nucl. Phys. A638, 511C (1998); M. Alford, K. Rajagopal and F. Wilczek, Nucl. Phys. A638, 515C (1998), hep-ph/9802284; K. Rajagopal, Prog. Theor. Phys. Suppl. 131, 619 (1998), hep-ph/9803341; J. Berges and K. Rajagopal, Nucl. Phys. B538, 215 (1999), hep-ph/9804233; M. Alford, K. Rajagopal and F. Wilczek, Nucl. Phys. B537, 443 (1999), hep-ph/9804403; T. Schäfer, Nucl. Phys. A642, 45 (1998), nucl-th/9806064; S. Hands and S.E. Morrison [UKQCD Collaboration], Phys. Rev. D59, 116002 (1999), hep-lat/9807033; K. Rajagopal, Nucl. Phys. A642, 26 (1998), hep-ph/9807318; N. Evans, S.D.H. Hsu and M. Schwetz, Nucl. Phys. B551, 275 (1999), hep-ph/9808444; S. Morrison [UKQCD Collaboration], Nucl. Phys. Proc. Suppl. 73, 480 (1999), hep-lat/9809040; T. Schäfer and F. Wilczek, Phys. Lett. B450, 325 (1999), hep-ph/9810509; N. Evans, S.D.H. Hsu and M. Schwetz, Phys. Lett. B449, 281 (1999), hep-ph/9810514; R.D. Pisarski and D.H. Rischke, Phys. Rev. Lett. 83, 37 
(1999), nucl-th/9811104; K. Langfeld and M. Rho, hep-ph/9811227; T. Schäfer and F. Wilczek, Phys. Rev. Lett. 82, 3956 (1999), hep-ph/9811473; D.T. Son, Phys. Rev. D59, 094019 (1999), hep-ph/9812287; A. Chodos, H. Minakata and F. Cooper, Phys. Lett. B449, 260 (1999), hep-ph/9812305; J. Hosek, hep-ph/9812515; G.W. Carter and D. Diakonov, Phys. Rev. D60, 016004 (1999), hep-ph/9812445; D.K. Hong, hep-ph/9812510; S. Hands and S. Morrison, hep-lat/9902011; N.O. Agasian, B.O. Kerbikov and V.I. Shevchenko, hep-ph/9902335; R.D. Pisarski and D.H. Rischke, Phys. Rev. D60, 094013 (1999), nucl-th/9903023; T.M. Schwarz, S.P. Klevansky and G. Papp, Phys. Rev. C60, 055205 (1999), nucl-th/9903048; M. Alford, J. Berges and K. Rajagopal, hep-ph/9903502; T. Schäfer and F. Wilczek, Phys. Rev. D60, 074014 (1999), hep-ph/9903503; R. Rapp, T. Schäfer, E.V. Shuryak and M. Velkovsky, hep-ph/9904353; D. Blaschke, D.M. Sedrakian and K.M. Shahabasian, astro-ph/9904395; S. Hands and S. Morrison, hep-lat/9905021; G.W. Carter and D. Diakonov, hep-ph/9905465; E. Shuster and D.T. Son, hep-ph/9905448; A. Chodos, F. Cooper and H. Minakata, hep-ph/9905521; D.K. Hong, hep-ph/9905523; R.D. Pisarski and D.H. Rischke, nucl-th/9906050; D.K. Hong, V.A. Miransky, I.A. Shovkovy and L.C. Wijewardhana, hep-ph/9906478; T. Schäfer and F. Wilczek, Phys. Rev. D60, 114033 (1999), hep-ph/9906512; D.K. Hong, M. Rho and I. Zahed, hep-ph/9906551; M. Rho, nucl-th/9908015; R. Casalbuoni and R. Gatto, Phys. Lett. B464, 111 (1999), hep-ph/9908227; M. Alford, J. Berges and K. Rajagopal, hep-ph/9908235; W.E. Brown, J.T. Liu and H. Ren, hep-ph/9908248; E.V. Shuryak, hep-ph/9908290; S.D.H. Hsu and M. Schwetz, hep-ph/9908310; G.W. Carter and D. Diakonov, hep-ph/9908314; D. Blaschke, T. Klahn and D.N. Voskresensky, astro-ph/9908334; F. Wilczek, hep-ph/9908480; T. Schäfer, nucl-th/9909013; A. Chodos, F. Cooper, W. Mao, H. Minakata and A. Singh, hep-ph/9909296; R. Casalbuoni and R. Gatto, hep-ph/9909419; T. Schäfer, hep-ph/9909574; P.F. Bedaque, hep-ph/9910247; M. Alford, J. Berges and K. Rajagopal, hep-ph/9910254; B. Vanderheyden and A.D. Jackson, hep-ph/9910295; N. Evans, J. Hormuzdiar, S.D.H. Hsu, M. Schwetz, hep-ph/9910313; R.D. Pisarski and D.H. Rischke, nucl-th/9910056.

[3] A.W. Overhauser, Advances in Physics 27, 343-363 (1978).

[4] D.V. Deryagin, D.Y. Grigorev and V.A. Rubakov, Int. J. Mod. Phys. A7, 659-681 (1992).

[5] E. Shuster and D.T. Son, hep-ph/9905448.

[6] B.Y. Park, M. Rho, A. Wirzba and I. Zahed, hep-ph/9910347.

[7] D.K. Hong, M. Rho and I. Zahed, hep-ph/9906551, Phys. Lett. B in print.

[8] R. Casalbuoni and R. Gatto, Phys. Lett. B464, 111 (1999), hep-ph/9908227. 
[9] U.G. Meissner and I. Zahed, Z. Phys. A327, 5 (1987).

[10] R.D. Pisarski and D.H. Rischke, Phys. Rev. D60, 094013 (1999), nucl-th/9903023.

[11] R.D. Pisarski and D.H. Rischke, nucl-th/9907041.

[12] M. Le Bellac, Thermal Field Theory (Cambridge University Press, Cambridge 1996).

[13] I. Zahed and D. Zwanziger, hep-th/9905109; Phys. Rev. D in print.

[14] D.T. Son, Phys. Rev. D59, 094019 (1999), hep-ph/9812287.

[15] T. Schäfer and F. Wilczek, Phys. Rev. D60, 114033 (1999), hep-ph/9906512.

[16] M. Alford, K. Rajagopal and F. Wilczek, Nucl. Phys. B537, 443 (1999), hep-ph/9804403; T. Schäfer and F. Wilczek, Phys. Rev. Lett. 82, 3956 (1999), hep-ph/9811473.

[17] V. Bernard, U.G. Meissner and I. Zahed, Phys. Rev. D36, 819 (1987).

[18] M. Kirchbach and D.O. Riska, Nucl. Phys. A578, 511 (1994).

[19] V. Thorsson and A. Wirzba, Nucl. Phys. A589, 633 (1995), nucl-th/9502003.

[20] R.D. Pisarski and M. Tytgat, Phys. Rev. D54, 2989 (1996), hep-ph/9604404.

[21] R.D. Pisarski and D.H. Rischke, nucl-th/9907094.

[22] D. Son and M. Stephanov, hep-ph/9910491. 